\title{
Time Resolved Camera: The New Frontier of Imaging Devices
}

\author{
Salvatore Tudisco \\ INFN - Laboratori Nazionali del Sud, Catania \\ Italy
}

\section{Introduction}

Time resolved imaging up to the single photon sensitivity is one of the most ambitious and important goals of photonics. Currently there are no commercial devices able to provide both the information on position (imaging) and arrival time of photons emitted by weak and ultra-weak sources. Only few and very expensive devices (Intensified CCD, electron bombarded CCD etc.) are able to reach the single photon detection threshold together with the possibility to collect photons in small time window (up to few ns). Unfortunately such devices are not able to provide any information on the arrival time of photons, fundamental for the recent developments on ultra-fast, time correlated optical sensing techniques.

Fluorescence-based imaging (both single and multi-photon) is the research field that has most influenced the development of fast and sensitive optical detectors. Examples of techniques in this class include Förster resonance energy transfer (FRET) (Jares-Erijman et al., 2003), fluorescence lifetime imaging microscopy (FLIM) (Becker et al., 2006), and fluorescence correlation spectroscopy (FCS) (Schwille et al., 1999). The success of these techniques, particularly FLIM, derives from the ability to characterize an environment based on the time domain behaviour of certain fluorophores with high resolution in space domain. This characterization can be done today with high levels of accuracy in 3D with minimal interference from the surroundings and almost no dependence on fluorophore concentration.

Many others scientific areas like astronomy, biophysics, biomedicine, nuclear and plasma physics etc. can benefit from a time resolved imaging device; it can improve the actual detection limits providing physical information otherwise inaccessible.

In astronomy and astrophysical science, one of the toughest problems affecting groundbased telescopes is the presence of the atmosphere, which distorts the spherical wave-front, creating phase errors in the image-forming ray paths. Even at the best sites, ground-based telescopes observing at visible wavelengths cannot achieve an angular resolution in the visible better than telescopes of 10 to $20 \mathrm{~cm}$ diameter, because of atmospheric turbulence alone. The cause is random spatial and temporal wave-front perturbations induced by turbulence in various layers of the atmosphere; one of the principal reasons for flying the Hubble Space Telescope was to avoid this image smearing. In addition, image quality is affected by permanent manufacturing errors and by long timescale wave-front aberrations 
introduced by mechanical, thermal, and optical effects in the telescope, such as defocusing, decentring, or mirror deformations generated by their supporting devices.

Adaptive optics (AO) and Natural Guide Star (NGS) are the solutions to these problems: a deformable mirror is inserted in the light path of the telescope, and its control signal is based on measurement of the incoming wave-front, performed by a suitable high-sensitivity and time resolved imaging detector. NGS adaptive optics suffers from the fact that it is not always possible to find a suitable NGS close enough to the portion of sky under investigation. Normally only $1 \%$ of the sky has an available NGS for current AO systems. The most promising way to overcome the lack of sufficiently bright natural reference stars is the use of artificial reference stars, also referred to as laser guide stars (LGS). These are patches of light created by the back-scattering of pulsed laser light by sodium atoms in the high mesosphere, or in the case of Rayleigh LGS, by molecules and particles located in the low stratosphere. Such an artificial reference star can be created as close to the astronomical target as desired, if will be available a wave-front sensor measuring the LGS wave-front in order to correct the atmospheric aberrations on the target object.

In plasma science, even 60 years after the invention of the laser, we witness a rapid development of systems generating electromagnetic pulses with extreme parameters such as duration, wavelength, peak power, and focused intensity.

The employment of solid-state laser materials allows the generation and subsequent amplification of light pulses as short as a few optical cycles only. When combined with the technique of chirped pulse amplification - CPA (Strickland et al. 1985) where the laser pulses are temporally stretched and recompressed before and after their amplification, table-top laser systems reaching peak powers of several tens or hundreds of Terawatt $\left(1 \mathrm{TW}=10^{12} \mathrm{~W}\right)$ can be realized. At such huge intensities, the rapidly oscillating electric field of the laser pulse reaches peak values exceeding the atomic fields binding the electrons to the positively charged nucleus by several orders of magnitude. It is due to this fact that all kinds of matter when exposed to laser light shot under such extreme conditions are almost instantaneously ionized and a plasma - sometimes called the "4th state of matter" - is formed. Within such plasma, the interaction between the charged constituents mediated by the long-range Coulomb interaction governs the behaviour and the evolution of the plasma. This gives rise to a large magnitude of effects that makes the generation and application of plasmas a fascinating field of current research in physics.

In this context, the use of a time resolved imaging devices to get information on the spatial and temporal evolution of laser generated plasma is fundamental to improve the actual level of knowledge.

Thus far, in many time-resolved and/or high-sensitivity applications the detectors of choice have been photomultiplier tubes (PMTs) and multichannel plates (MCPs) (McPhate et al. 2005). While these devices can reach time uncertainties of a few tens of picoseconds (MCPs), usually are bulky, fragile, sensitive to electromagnetic disturbances (especially PMTs) and mechanical vibrations, require high supply voltages $(2-3 \mathrm{kV})$ and are costly devices, particularly the high-performance models. Moreover multi-pixel images are not possible without bulky setups and expensive equipment. Thus, high sensitivity and/or timeresolved imaging has been relegated to applications requiring important investments for optical and detector equipment. 
A number of solid-state solutions have been proposed as a replacement of MCPs and PMTs using conventional imaging processes. The challenge, though, has been to meet single photon sensitivity and low timing uncertainty. To address the sensitivity problem, cooled and/or intensified CCDs (Etoh et al. 2005) and ultra-low-noise CMOS APS architectures (Kawai et al. 2005) have been proposed. Multiplication of photo-generated charges by impact ionization has also been used in CCDs (Hynecek et al. 2001).

As an alternative to PMTs and MCPs, researchers have turned to solid-state photon counters based on avalanche photodiodes (APDs). In the last four decades, solid-state multiplication based photo-detectors have gradually evolved from relatively crude devices to the sophistication of today. Semiconductor APDs have the typical advantages of solid state devices (small size, low bias voltage, low power consumption, ruggedness and reliability, suitability to build integrated systems, etc.). Their quantum detection efficiency is inherently higher, particularly in the red and near infrared range. In APDs operating in linear mode the internal gain is not sufficient or barely sufficient to detect single photons. However, single photons can be efficiently detected by avalanche diodes operating in Geiger-mode, known as single-photon avalanche diodes (SPADs). Almost every imaging technology has one photo-detector device and the range of implementations is quite wide. In this context, SPADs have recently attracted significant interest thanks to their relative simplicity and ease of fabrication.

\section{Single photon avalanche diodes}

In the last decades, the possibility to build a silicon photo-sensor suitable for single photon counting applications was investigated by several research teams (Cova et al. 1983 and references therein). The original idea, firstly proposed by R.J. Mc Intyre since the 1960s, was to implement a semiconductor photodiode with characteristics suitable for the triggered avalanche operation mode, known as Geiger mode of operation, and therefore able to detect single photons.

Carriers generated by the absorption of a photon in the $\mathrm{p}-\mathrm{n}$ junction, are multiplied by impact ionization thus producing an avalanche. APDs can reach timing uncertainties as low as a few tens of picoseconds thanks to the speed at which an avalanche evolves from the initial carrier pair forming in the multiplication region. An APD is implemented as photodiode reverse biased near or above breakdown, where it exhibits optical gains greater than one. When an APD is biased below breakdown it is known as proportional or linear APD. It can be used to detect clusters of photons and to determine their energy. When biased above breakdown, the optical gain becomes virtually infinite (see fig. 1). Thus, with relatively simple ancillary electronics, the APD becomes capable of detecting single photons. The APD operating in this regime is called single-photon avalanche diode - SPAD. If the primary carrier is photo-generated, the fast leading edge of the avalanche pulse marks the arrival time of the detected photon. After the avalanche is triggered, the current keeps flowing until the avalanche is quenched by lowering the bias voltage down to breakdown voltage or below. The bias voltage is then restored in order to detect another photon. This operation requires a suitable circuit that is usually referred to as a quenching circuit.

Individual detectors and detector arrays based on the SPAD technology have received renewed interest in recent years due to the versatility of their applications. 
There are two main lines of research in silicon SPADs: one that advocates the use of highly optimized processes to boost performance and one that proposes to adapt SPAD design to existing processes to reduce cost and to maximize miniaturization. Both approaches have advantages and drawbacks.

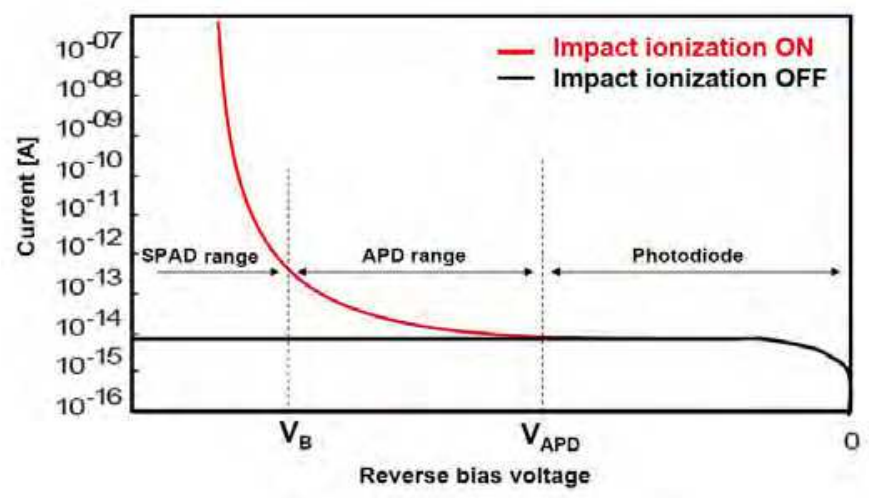

Fig. 1. Typical multiplication regions of reverse polarization junction.

The basic goals of the SPAD fabrication technology are to: (i) keep low the dark counting rate; (ii) keep low the afterpulsing probability; (iii) make uniform the electric field over the whole active area in order to have a photo-detection efficiency (PDE) independent from the absorption position; (iv) keep low the photo-generation of carriers outside the multiplication region in order to minimize the time uncertainty.

As it happens in PMTs, thermal generation effects produce current pulses even in the absence of illumination, and the Poissonian fluctuation of these dark counts represents the internal noise source of the detector. The dark pulses are due to carriers thermally generated in the SPAD junction, so that the count rate increases with the temperature, as does the dark current in ordinary photodiodes. The rate also increases with the excess bias voltage (EBV, which is the voltage over the breakdown) because of two effects: $i$ ) field-assisted enhancement of the emission rate from generation centers (Hurkx et al. 1996) and ii) increase of the avalanche triggering probability (Oldham et al. 1972).

The SPAD count rate includes also secondary pulses due to afterpulsing effects that in the case of dark count may strongly enhance the total measured rate.

During the avalanche some carriers are captured by deep levels in the junction depletion layer and subsequently released with a statistically fluctuating delay, whose mean value depends on the deep levels actually involved (Cova et al. 1991). Released carriers can retrigger the avalanche, generating afterpulses correlated with a previous avalanche pulse. The number of carriers captured during an avalanche pulse increases with the total number of carriers crossing the junction, that is, with the total charge of the avalanche pulse. Therefore afterpulsing increases with the delay of avalanche quenching and with the current intensity, which is proportional to the EBV (usually dictated by photon detection efficiency or time resolution requirements, or both (Cova et al. 1996)) so that the trapped charge per pulse first has to be minimized by minimizing the quenching delay. The situation is even 
more disadvantageous when the detector is cooled to reduce the dark-counting rate, since the release from trapping states becomes slower.

In conclusion, a really suitable technology for producing SPADs must not only reduce the generation and regeneration centers to very low concentration level, but also eliminate trapping level or at least minimize their concentration. The technological challenge is to design a process with such characteristics and still compatible with standard microelectronic industry processes.

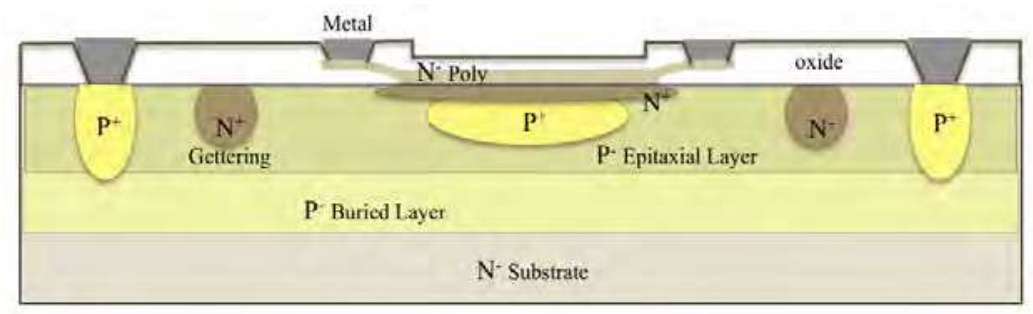

Fig. 2. Junction scheme of SPAD device

\subsection{Fabrication and structure design}

There exist several implementation styles for APDs, of which two are the most used. In the first style, known as reach-through APD, one builds a $p+-\pi-p-n$ structure where $\pi$ denotes very lightly $p$-doped (McIntyre, 1985). When reverse biased, the depletion region extends from the cathode to the anode. Thus, the multiplication region is deep in the $p / n+$ junction. Due to the depth of the multiplication region, this device is indicated for absorption of red and NIR photons up $1.1 \mu \mathrm{m}$ (for silicon). Since the photoelectrons drift until the multiplication region, a larger timing uncertainty is generally observed.

The second implementation style is compatible with planar CMOS processes and it involves a shallow or medium depth $p$ or $n$ layer to form high-voltage $p n$ junctions. Cova and others have investigated devices designed in this style since the 1970s, yielding a number of structures (Cova et al. 1981). All these structures have in common a $p n$ junction and a zone designed to prevent premature edge breakdown. An example of the early structures is reported in the work of Zappa et al. (1997) $n+/ p+$ enrichment in p-substrate was used, while premature edge breakdown was prevented by confining $p+$ enrichment in the centre of the device.

More recently, many authors have developed APDs, both in linear and Geiger mode, using dedicated planar and non planar processes, achieving superior performance in terms of sensitivity and noise (Kindt 1999).

The main disadvantage of using dedicated processes is generally the lack of libraries that can support complex functionalities and deep-submicrometre feature sizes, thus limiting array sizes. An interesting alternative is the use of a hybrid approach whereby the APD array and ancillary electronics are implemented in two different processes, each optimized for APD performance and speed, respectively. If the ancillary electronics is implemented in CMOS, high degrees of miniaturization are possible. The price to pay is increased 
fabrication complexity. The integration of SPADs in a low-cost CMOS process became feasible since 2003 (Sciacca et. al 2003).

An example of latest developed structures is shown in figure 2 (Sciacca et. al 2003). The $n p$ junction has an $n+$ shallow diffusion and a controlled Boron enrichment diffusion in the central zone of it. With respect to the outer abrupt $n p$ junction, the higher $p+$ doping concentration reduces the breakdown voltage in the central zone, which is the active area.

The process starts with a $\mathrm{Si}<100>n$ - substrate on which is grew a boron doped epitaxial layer with a $p+$ buried layer and with a $p$ - doped layer. The reason to form a buried $p n$ junction is twofold. First, the detector time-response is improved because the effect of photogenerated carriers diffusing in the undepleted region is reduced (G. Ripamonti et al. 1985). Second, isolation with the substrate is introduced and makes possible the monolithic integration of various SPADs and other devices and circuits. The $p+$ buried layer is necessary to reduce the series resistance of the device.

The $p$ - layer must be thin enough to limit the photo-carrier diffusion effect above mentioned. A good trade off has to be found for this thickness, because if it is made too thin the edge breakdown occurs at a voltage not much higher than the breakdown voltage of the active area. The $p+$ sinkers are then created with a high-dose boron implantation step, in order to reduce the contact resistance of the anode and provide a low resistance path to the avalanche current.

The next step, a local gettering process, is a key step in the process because it guarantees a uniform defect concentration over the volume. At this point a heavy $\mathrm{POCl}_{3}$ diffusion through an oxide mask is made on the topside of the wafer close to the device active area. Heavy phosphorus diffusions are well known to be responsible for transition metal gettering. Unfortunately, the well-known phosphorous predeposition on the backside of the wafer is not able to getter the distant active area of the device because metal diffusers ( $\mathrm{Pt}$, $\mathrm{Au}, \mathrm{Ti}$ ) diffuse too slowly during the final anneal. For this reason, if the gettering sites are created suitably close to the active region, a major improvement on dark counting rate is observed. The next step is the $p+$ enrichment diffusion obtained with a low energy boron implantation, producing a concentration peak, followed by a high temperature anneal and drive in (Lacaita et al. 1989).

The first generation of devices was fabricated with a deposited polysilicon cathode doped by Arsenic implantation and diffusion. The As+ ion implantation energy was carefully calculated in order to damage as little as possible the active area of the device; nevertheless, devices with very high dark-counting rate resulted. A remarkable improvement was obtained in the second generation by doping in situ the polysilicon. Further improvement was achieved in the third generation by accurately designing a Rapid Thermal Anneal to create a precisely controlled shallow Arsenic diffusion below the polysilicon in the $p$ epilayer.

An important issue for the SPAD quality is the uniformity of the electric field over the active area. If the electric field is not uniform, the PDE of the device becomes dependent on the absorption position over the active area. The lower the electric field the lower the PDE, the worst case being when the electric field is lower than the breakdown value. 


\subsection{Quenching strategy}

After the avalanche is triggered, the current keeps flowing until the avalanche is quenched by lowering the bias voltage down to breakdown voltage or below. After a dead-time, the operative voltage must be restored in order to make the SPAD able to detect another photon. This operation requires a suitable electronics with the following tasks: (i) it senses the leading edge of the avalanche current; (ii) it generates a standard output pulse, synchronous with the current onset; (iii) it quenches the avalanche by lowering the bias below the breakdown voltage; (iv) it restores the photodiode voltage to the operating level. This circuit is usually referred to as quenching circuit. The two main quenching strategies are: passive and active (Cova et al. 1996). Recently, very promising results were obtained by using also a mixed approach (Mingguo et al. 2008).

In passive quenching the avalanche current itself is used to drop the voltage across the diode. This is generally accomplished via a ballast resistor $\mathrm{R}_{\mathrm{L}}$ (normally few hundreds $\mathrm{k} \Omega$ ) placed on the anode or the cathode of the diode as shown in figure 3 . The detection of the avalanche can be accomplished by measuring the current across a low resistivity path $R_{\text {s. }}$ Pulse shaping may be performed using a discriminator.

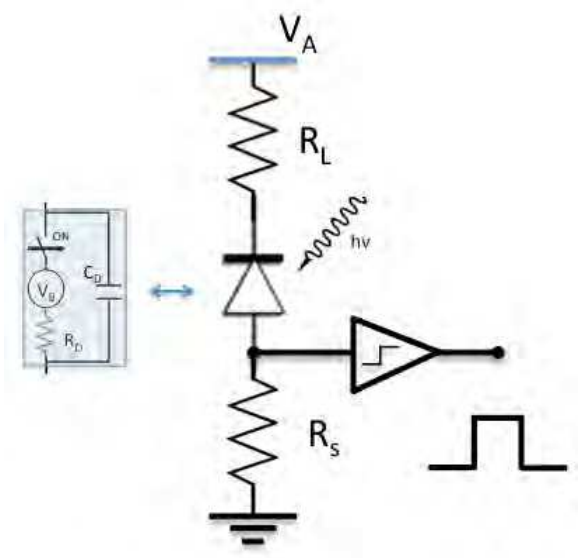

Fig. 3. Schematic representation of a passive quenching circuit. In the left part is shown the equivalent circuit of a SPAD detector.

Today modern technology gives also the possibility to produce SPAD detectors with integrate quenching mechanism based on a Metal-Resistor-Semiconductor structure. Precise resistive elements (or using the non-linear characteristics of a biased PMOS or NMOS, Niclass et al. 2005) are embedded for each individual micro-cell of the array and provide effective feedback for stabilization and quenching of the avalanche process also reducing the values of the stray capacitance from cathode to ground.

A circuit model, which emulates the evolution of the signal of a SPAD was developed in the 1960s to describe the behaviour of micro-plasma instability in silicon (McIntyre 1961). According to this model, the pre-breakdown state can be represented as a capacitance (junction capacitance, $C_{D}$ ) in series with the quenching resistor. Referring to Fig. 3(left), this 
state corresponds to the switch in the OFF condition. In steady state, the capacitance is charged at operating voltage $V_{A}>V_{B}$ where $V_{B}$ is the breakdown voltage.

When a carrier traverses the high-field region, there is a certain probability, known as turn on probability, to initiate an avalanche discharge. If this happens, the new state of the system can be modelled adding to the circuit a voltage source $V_{B}$ with a series resistor $R_{D}$ in parallel to the diode capacitance (switch closed in Fig. 3(left)). $R_{D}$ includes both the resistance of the neutral regions inside the silicon as well as the space charge resistance. $C_{D}$, originally charged at $\mathrm{V}_{\mathrm{A}}$, discharges through the series resistance down to the breakdown voltage with a time constant $\tau_{D}$ given by the product $R_{D} C_{D}$. It should be noted that the discharge current is initially limited by the build up of the avalanche process which can take some hundreds of ps.

As the voltage on $C_{D}$ decreases, the current flowing through the quenching resistance, and as a consequence through the diode, tends to the asymptotic value of $\left(V_{A}-V_{B}\right) /\left(R_{L}+R_{D}\right)$. In this final phase, if $R_{L}$ is high enough, the diode current is so low that a statistical fluctuation brings the instantaneous number of carriers flowing through the high-field region to zero, quenching the avalanche. The probability of such a fluctuation (turnoff probability) becomes significant when the diode current is below 10-20 mA (defined as latching current). The average time needed to stop the avalanche, when this condition is satisfied, is in the order of $1 \mathrm{~ns}$. The latching current poses a strict limit on the lower value of $\mathrm{R}_{\mathrm{L}}$ to some hundreds of $\mathrm{k} \Omega$. As the avalanche process is terminated, the switch is again open and the circuit is in its initial configuration. The capacitance charged at $\mathrm{V}_{\mathrm{B}}$, starts recharging to the bias voltage with a time constant $C_{D} R_{L}$, and the device becomes ready to detect the arrival of a new photon.

A photon that arrives during the very first part of recovery is almost certainly lost, since the avalanche triggering probability is almost negligible. Instead, subsequent photons have a progressively higher probability to trigger the SPAD. Unfortunately, SPAD triggering during recovery transition has mainly two deleterious effects due to the time-varying excess bias voltage.

Pulses having amplitude lower than the threshold of the discriminator are not sensed. Significant count losses are expected at higher counting rates. As a matter of fact, after each ignition, the detector has a dead-time which is not well-defined.

Time resolution is degraded for two reasons: the intrinsic time resolution of the SPAD is impaired when excess bias is reduced; additional jitter is introduced because pulses with different amplitudes cross the comparator threshold at different times.

In order to avoid the highlighted drawbacks of passive quenching circuit (PQC) and fully exploit the intrinsic performance of SPADs, a new approach was devised (Cova et al. 1996). The basic idea was to sense the rise of the avalanche pulse and react back on the SPAD, by forcing the quenching and reset transitions in short (few nanoseconds) times, with a controlled bias-voltage source. This approach was called active-quenching circuit (AQC) the literature on active quenching is extensive.

The simplest solution provides that the discriminator triggers also a driving stage that applies a quenching pulse, synchronous with the avalanche triggering and with a very low jitter. In order to quench the avalanche, the quenching pulse must be high enough to reduce 
the diode voltage below $V_{\text {B. }}$. The detector is then kept off for a well-controlled hold-off time, at the end of which the driver swiftly restores the SPAD bias voltage to the operating level in order to be ready to detect another photon. During the reset transition, since spurious couplings and reflections could retrigger the discriminator, it can be latched off for the whole reset duration.

In this way the avalanche current intensity is kept constant due to the low-impedance of the driver biasing the detector, also the duration of the avalanche current pulse is constant and depends on the time taken by the avalanche signal to travel from the SPAD to the AQC, forth and back, and on the slope of the driver quenching pulse. By reducing this loop time, the afterpulsing will be limited, since the number of trapped carriers is linearly proportional to the avalanche pulse duration. Moreover, by using ACQ, the hold-off time is easily adjustable and both quenching and reset transitions are fast (tens of nanoseconds), thus minimizing the probability of non-standard avalanche triggering during the recovery transition. Nevertheless, recent works (Neri et al. 2010) demonstrates that with passive quenching strategy (by using hybrid Dead-Time models) it is also possible to evaluate the real amount of incident photon rate up to $10^{7}-10^{8} \mathrm{cps}$.

In conclusion, even if ACQ are attractive, they usually require an extra complexity to a pixel, hindering the miniaturization, fundamental for imaging aim.

\subsection{Performance and measurement techniques}

In this section and in next, the attention will focus on SPADs with integrate quenching resistor. In contrast to APDs, to establish the performances are necessary a new set of parameters and measurement techniques.

A primary "static" characterization is made on-wafer by using temperature-controlled probe stations and semiconductor parameter analysers. By working in dark condition the SPAD reverse/forward I-V characteristics can be determined, with and without quenching resistor and as a function of the temperature. In this way we get information about leakage current, breakdown voltage, quenching resistor and hence on the overall device performance. For example the leakage current is linked to the generation of electrical carriers both in the bulk as well as on the surface depleted region around the junction. Then, low values of leakage current can be interpreted as the first evidence of the low defectivity of the diode and then of the low dark counting rate. The figure 4 reports the I-V characteristics of ST-Microelectronics SPAD (Mazzillo et al. 2008).

The operation of a SPAD with integrated quenching resistor can be easily investigated by measuring the current across a low resistivity path $\mathrm{R}_{\mathrm{S}}$ ("dynamic" characterization). In figure $5 \mathrm{a}$ is reported the pulse of a single dark count event observed on a FBK SPAD.

The fast leading edge (rise time) is governed by the time that avalanche takes to spread all over the diode active area. The slower exponential decay (discharge time) as previous mentioned is instead determined by a constant time given by the product of the diode internal resistance (few $\mathrm{k} \Omega$ ) and the sum of parasitic and internal diode capacitances. After the breakdown current has been quenched, the diode will slowly recharge through the quenching resistor (see fig.5b). 

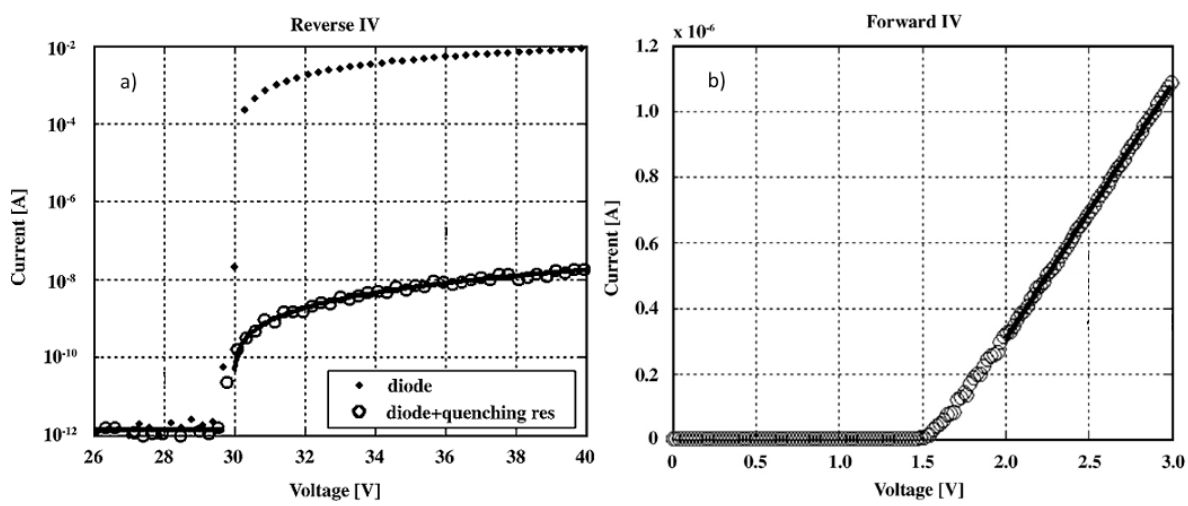

Fig. 4. I-V characteristics for a square shaped ( $30 \mu \mathrm{m}$ side) ST-Microelectronics SPAD; $a)$ reverse; $b$ ) forward.

Total charge and device gain can be extracted by integrating the pulse, converted into a current signal. The junction capacitance can be evaluated estimating the slope of the chargeEVB characteristic.

The Dark Noise is a function of EBV, temperature and detector area. Commonly it may vary from a minimum of tens of hertz to a few kilohertz and is a superposition of dark count and afterpulsing rate. In figure $5 \mathrm{~b}$ the oscilloscope was triggered on a SPAD Geiger pulse (either dark count or afterpulsing event), while the device was kept in dark condition. The time delay between the leading edges of the reference pulse and a consecutive pulse, which can be due to dark count or afterpulsing, was measured. Then the average delay between two consecutive pulses was obtained. The inverse of this value represents the dark noise rate of the device.

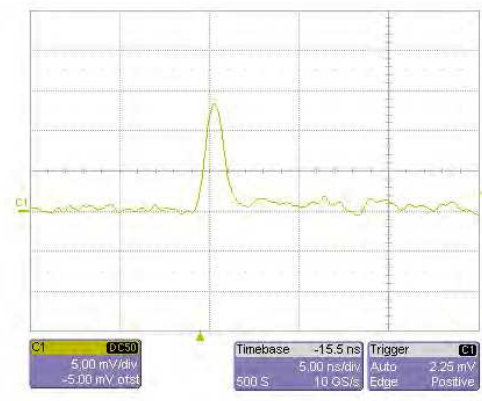

a) b)

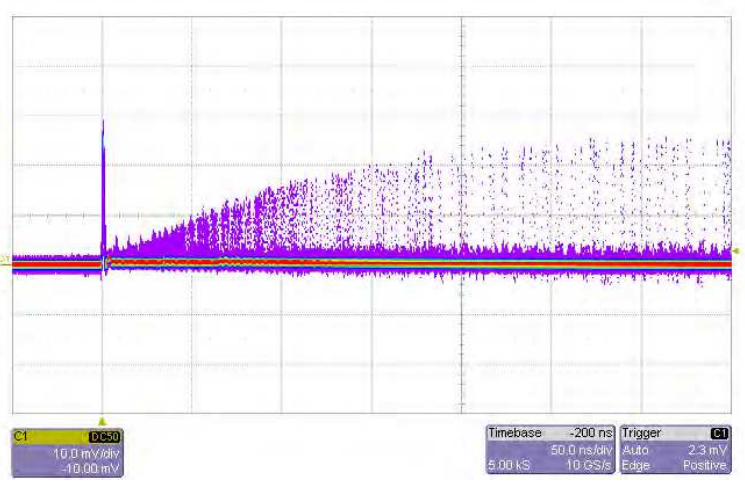

Fig. 5. Dynamic characterization of a circular FBK-IRST SPAD (30 $\mu \mathrm{m}$ diameter). The signals in $a$ ) and $b$ ) correspond to dark events when the device is respectively biased at 10 and $20 \%$ of EBV. In $a$ ) is displayed the structure of a single pulse; $b$ ) reports the afterpulses distribution through a persistence image. 
The distribution of the Afterpulsing events, as a function of the temperature and EBV, can be obtained by building the histogram of the time delay between two consecutive pulses within a time window of some hundreds of ns, being the probability of two independent dark counts events in such a time window negligible (the average time scale between two uncorrelated dark events is of the of the order of $\mathrm{ms}$ ). The distribution is then normalized to the total number of collected events. The histogram is bell-shaped (Mazzillo et al. 2008) as a convolution of the exponentially decreasing probability of releasing a carrier from a trap and the exponentially increasing EBV due to the recharging phase of the depletion layer. The probability that a released carrier can trigger an avalanche event increases with time because during the recharging phase the voltage at the depletion layer approaches exponentially to the bias voltage. On the other hand the probability of release of the captured carriers decreases very quickly with time since this phenomenon is mainly due to traps that are located near the conduction and valence band edges. The afterpulsing probability is commonly a few percent.

The Photon Detection Efficiency (PDE) of SPAD detector is given by the product of two parameters: the quantum efficiency $(\mathrm{QE})$ and the avalanche triggering probability (PT).

The QE represents the probability for a photon to generate an $e-h$ pair in the active thickness of the device. It is given by the product of two factors: the transmittance of the dielectric layer on top of the silicon surface and the internal QE. Both are wavelength dependent. The former can be maximized, by implementing an anti-reflective coating. The second term represents the probability for a photon that has passed the dielectric layer to generate an $e-h$ pair in the active thickness. In a conventional $n+/ p / p+$ diode, the active layer is roughly limited on top by the undepleted $n+$ layer, whereas on the bottom by the $p+$ layer used for the ohmic contact or by the highly doped substrate in case of epitaxial substrates. Indeed, when a pair is generated in those regions, there is a high probability for the electron and hole to recombine due to Auger or Shockley-Read-Hall processes (Tyagi 1991). For short wavelengths, the problem is focused in the top layer. As an example, a 420nm light is almost totally absorbed in the first $500 \mathrm{~nm}$ of silicon, which, for non-optimized fabrication processes, is usually well inside the undepleted layer.

There is a finite probability for a carrier to initiate an avalanche when passing through a high-field region. In case of a photogeneration event, two carriers are created travelling in opposite directions. Both contribute to the $P T$ that can be evaluated from the following expression: $P T=P e+P h-P e P h$. Where $P e$ and $P h$ are the electron and hole breakdown initiation probabilities. These terms can be calculated as a function of the generation position by solving two differential equations involving the carrier ionization rates. $\mathrm{Pe}$ and $\mathrm{Ph}$ depend on the impact ionization rates of electrons $\left(\alpha_{n}\right)$ and holes $\left(\alpha_{p}\right)$, respectively. These parameters are not well determined yet, and large discrepancies exist among the values extracted from the various models (Grant et al. 1973). Anyway, despite the differences in absolute values, some features are well established: (i) both coefficients increase with the electric field, (ii) the electron has an ionization rate higher than the hole (is about twice $\alpha_{\mathrm{p}}$ ), and (iii) their difference decreases with increasing fields.

This behaviour is reflected in the probabilities $P e$ and $P h$. Thus, to maximize the triggering probability: (i) the photogeneration should happen in the $\mathrm{p}$ side of the junction in order for the electrons to pass the whole high field zone, and (ii) the bias voltage should be as high as possible. 

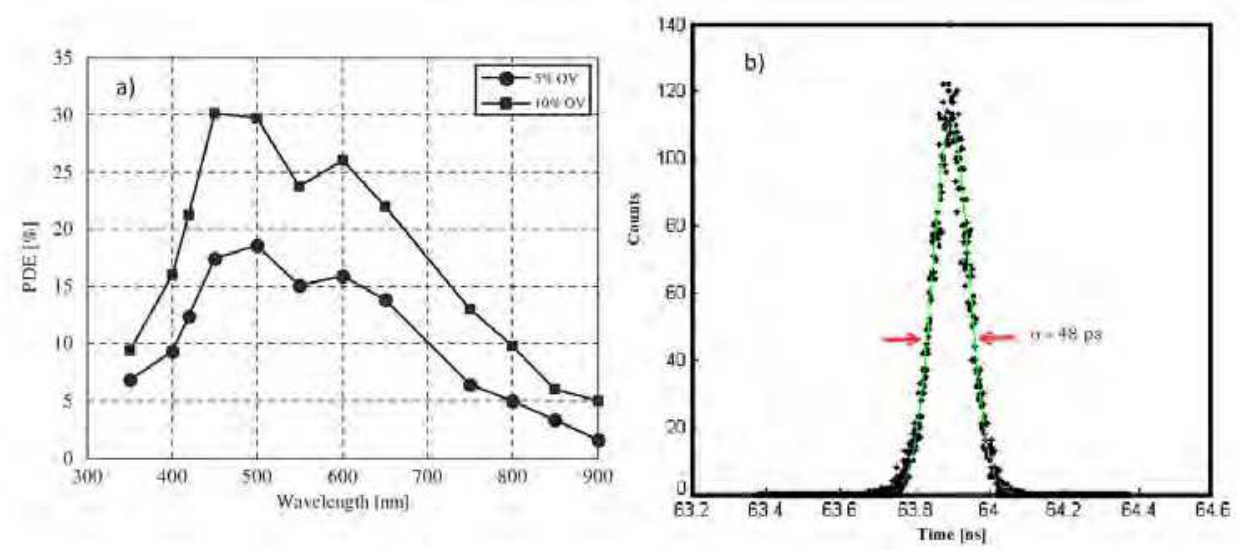

Fig. 6. a) PDE of ST-Microelectronics SPAD at 5 and $10 \%$ of EBV; $b$ ) Photon timing of passively quenched ST-Microelectronics SPAD.

The measurement principle adopted for the evaluation SPAD PDE is a direct comparison with a calibrated photodiode that receives the same photon flux of SPAD. An example of experimental set-up where, with high level of accuracy, are possible PDE measurements is discussed by Bonanno et al. 2005. In figure 6a is reported the typical PDE measured for the ST-Microelectronic devices.

The SPAD performance in Photon Timing is characterized by its time resolution curve, which is the statistical distribution of the delay between the true arrival time of the photon and the measured time, marked by the onset of the avalanche current pulse. These can be obtained by means of a time-correlated photon counting apparatus (Privitera at al. 2008), where optical pulses with short time duration (less than $30-40 \mathrm{ps}$ ) are used to illuminate the devices. After a careful check that the contribution of the laser pulse and the eventual electronic jitter to the SPAD response are negligible, by using a digital oscilloscope it is possible to build the histogram of the time delay between the laser reference pulse and the SPAD pulse.

In figure $5 \mathrm{~b}$ is reported the time resolution curve obtained by using laser pulses of $408 \mathrm{~nm}$ wavelengths, 35 ps FWHM and $1 \mathrm{kHz}$ repetition rate, on an ST-Microelectronic SPAD. It is consistent with the typical values reported in the literature for other devices.

\section{Bidimensional arrays of SPADs}

As discussed in the previous sections, SPAD arrays fabricated by standard planar silicon production processing, are at the moment considered an interesting topic of research because many photonic applications, not only scientific, would become available at much more reasonable cost.

The main requirement in the array fabrication is to guarantee electrical and optical isolation among pixels. In fact, each pixel must be operated independently from the others, independently on neighboring pixels and without disturbing them. 
A typical way to achieve electrical isolation in silicon planar technology is to employ isolation diffusion, i.e. a thus electrically isolating the epitaxial layer, of the opposite doping sufficiently deep diffusion that reaches the substrate of the same doping type, type, as shown in Figure 7. However, the hardest problem to solve in SPAD array design is optical coupling. This effect is peculiar to the above-breakdown operation of SPADs.

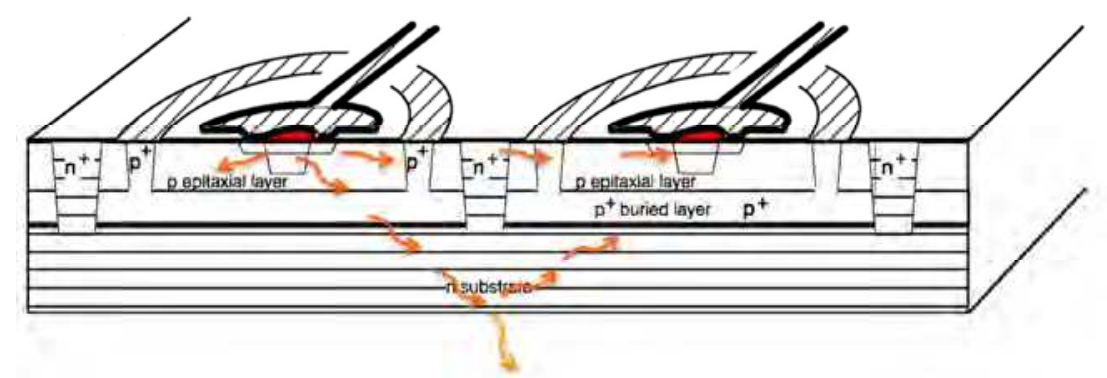

Fig. 7. Optical cross-talk representation between two neighbouring devices. The avalanche current in one pixel can trigger the process in surrounding pixels, due to photons emitted by hot carriers.

During the avalanche process each ionization impact causes high deceleration in electron drift, so the Bremsstrahlung process enables a secondary photons production inside the junction (the emission probability is estimated in $10^{-5}$ photons per carrier crossing the junction).

The optical cross talk is then activated by the emission of secondary photons, that can travel along the silicon bulk, transparent to the optical wavelength, and cross another diode that is triggering a correlated avalanche (Fig. 7). The time delay between the two produced signals is less than the measurable one and induced signals cannot be distinguished from the generated one. A further contribute to the optical cross talk was found in the indirect optical pat enabled by the optical reflection by the back part of the device.

In order to avoid the optical cross-talk, it is possible design and fabricate arrays that are optically and electrically isolated by deep thin trench technology. The trench process starts with a vertical etch (about $10 \mu \mathrm{m}$ deep and $1 \mu \mathrm{m}$ large), a subsequent oxide deposition for complete electrical isolation. The process continues with tungsten fill to avoid optical crosstalk and ends with planarisation.

Nevertheless, all the cross-talk contributions previous mentioned, become important when the density of implemented elements is higher and the distances between neighbouring devices are smaller as required by imaging applications or by silicon PhotoMultiplier technology. They influence the total Dark counting rate of the device.

\subsection{Silicon photomultiplier concept}

A photomultiplier based on the silicon technology represents the new frontier of photodetection. SPADs integrated on the same substrate, with a common read-out, could satisfy such expectations. The main limitation of a single diode working in Gaiger mode is 

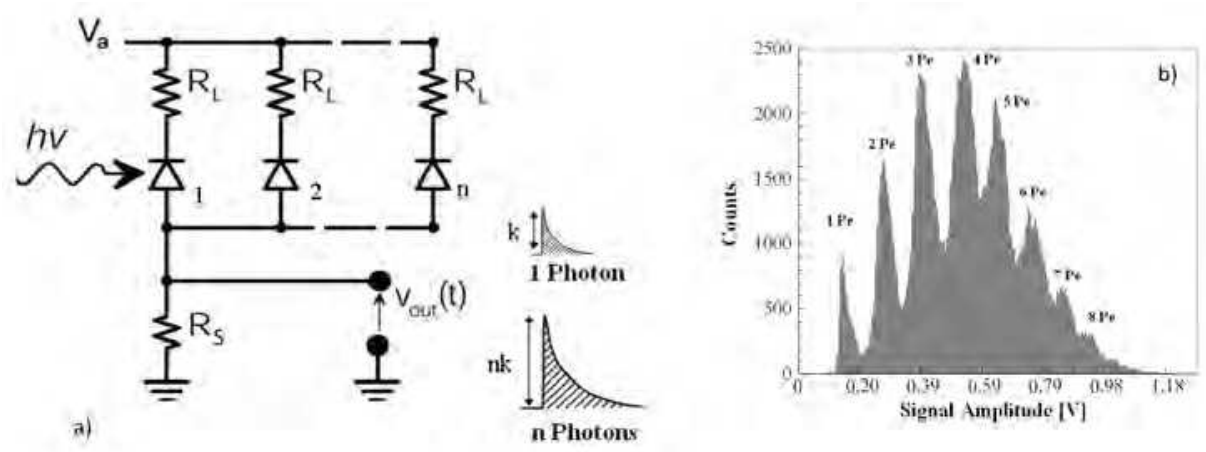

Fig. 8. a) Schematic representation of SiPM working principle. The amplitude of the out signals is proportional to the number of detected photons. In $b$ ) is reported the distribution.

that the output signal is the same regardless of the number of interacting photons. In order to partially overcome this limitation, the diode can be segmented in tiny micro-cells (each working in Gaiger mode) connected in parallel to a single output. Each element, when activated by a photon, gives the same current response, so that the output signal is proportional to the number of cells hit by a photon. The number of elements composing the device limits the dynamic range, and the probability that two or more photons hit the same micro-cell depends on the size of the micro-cell itself. This structure is called Silicon PhotoMultiplier (SiPM). Some of the advantages offered by the solid-state solution are: insensitivity to magnetic fields, ruggedness, compactness, low operating voltage and long lifespan. In addition, this technology facilitates the interconnection between the detector and the read-out electronics.

An interesting application of SiPMs is the detection of the light emitted by scintillators. Among the various types of scintillators, particular attention has recently been given to lutetium oxyorthosilicate (LSO) for its high light yield, short decay time and relatively good mechanical properties. These features are extremely useful, for example, in positron emission tomography (PET).

\subsection{Image sensor}

The identification of the fired pixel and the time at which the event occurred are the fundamental requirements in order to realize the time resolved imaging. These two features are almost simultaneously incompatible with the traditional reading techniques (CCD etc.). High-definition images require a large number of pixels and reading techniques designed to minimize the number of connections. On the other hand, these are almost incompatible with the information on the photon arrival time.

To avoid missing photon counts, a counter should be used for each pixel. However, large counters are not desirable due to the fill factor loss and/or extra time required to perform a complete readout of the contents of the chip. A possible solution to this problem is to access every pixel independently but sequentially using a digital random access scheme (Niclass et al. 2005). 
In low light level applications one can use an event-driven readout, where the detector initiates and drives a column-wise detection process directly (Niclass et al. 2006). The drawback of this approach is that multiple photons cannot be detected simultaneously on the same column. In addition, the bandwidth of the column readout mechanism limits saturation levels of the entire column. These limitations are not problematic if the expected photon flux hitting the sensor is low.

An alternative approach for no low light level situations is the use of a latchless pipeline scheme. In this approach, the absorption of a photon causes the SPAD to inject a digital signal into a delay line that is then read externally. The timing of all injected pulses is evaluated so as to derive the time of arrival of the photon and the pixel of origin. This method allows detection of photons simultaneously over a column even though some restrictions apply on the timing of the optical set up. Note that in this design an effective gating mechanism is necessary to prevent photons detected outside a certain time window being interpreted as originating in a pixel other than the one responsible for that detection. The pixel access problem can be overcome if the photon time of arrival is performed in situ, i.e. on the pixel itself.

The main problem of the all discussed solutions is the physical size of the on-board ancillary circuitries (digital signal extractor, counters, memory elements, buffer readout, etc.), which drastically decrees the fill factor and increase the complexity of the chip manufacturing.

The alternative approach is to bring out the chip all the readout circuitries in order to increase the fill factor, simplify the matrix design and the manufacturing process (Tudisco et al. 2009).

A cross-wire readout scheme could be adopted to access every pixel independently; the avalanche signal of each diode is twist extracted from two contacts, these are shared one for all diodes of the same row and the other for all diodes of the same column. Figure 9 reports the scheme of two possible configurations. In such a way it is possible to deduce the hit position with a readout complexity which grows as $2 n$ instead of $n^{2}$.

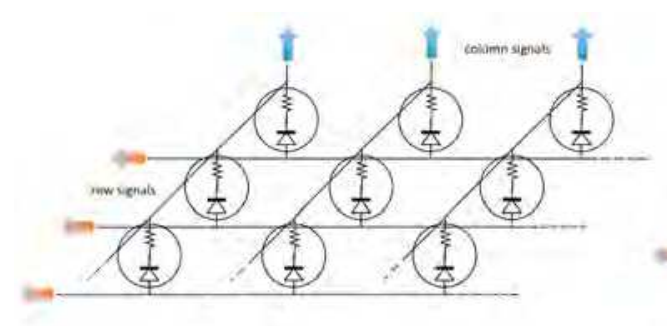

a)

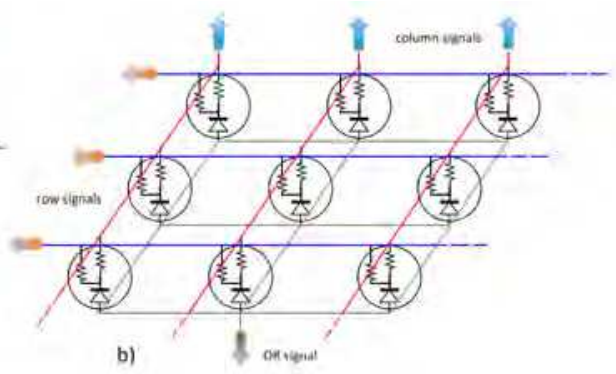

b)

Fig. 9. Cross-wire readout scheme. In $a$ ) the avalanche signal of each pixel is collected from both side of the junction. In $b$ ) each pixel has two quenching resistors and a common anode contact.

If one of the devices "fires" in response to light applied to that device, it will generate a current pulse in one of corresponding row and column outputs. This allows the specific device to be determined unambiguously by its unique row-column signature. 
The only real limitation of this spatial recognition approach is the uncertainty in the simultaneous detection of two photons. This uncertainty comes from events tagging and is related to the time jitter of row and column signals.

In the configuration of figure 9a the avalanche signal of each pixel is collected from both side of the junction, anode and cathode while the solution of figure $9 \mathrm{~b}$ requires the integration of two quenching resistors for each diode, with advantages and disadvantages related to the existence of a common anode contact. The latter gives the possibility to collect the total OR-signal (like in SiPM configuration). Figure 10 shows the layout of some arrays made in collaboration with the FBK-IRST and currently under test and characterization.

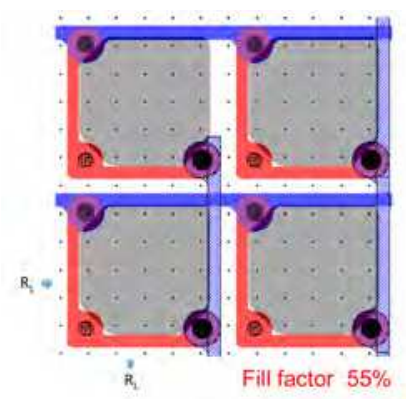

Fig. 10. Layout of FBK-IRST arrays.

Adopting the first solution was recently realised in collaboration with ST-Microelectronics an elementary first demonstrator of 10x10 elements (Tudisco et al. 2009).

The array layout has been designed with common anode and cathode bus. Metal strips used to contact the cathodes in each column are continuous while those used to contact the anodes in each row are interrupted in proximity of each cathode metal bus in order to avoid short-circuits with cathode metal strips. A high-doped silicon underpass guaranties the conductivity. The operating conditions were assured by the use of a single external quenching resistor for row (or column).

In such simplified configuration, respect to the figure $9 \mathrm{a}$, the main disadvantage is the quenching of all devices in the row (or column) join to the slow recharge time coming from to use of externals quenching resistor. Nevertheless it remains a valid concept device.

In order to realize a first working prototype of "Time Resolved Camera" (TRC), row and column signals can be treated by standard nuclear electronics. For example, by using commercial VME modules (Constant Fraction Discriminators and multi-hits Time to Digital Converters, fig. 11c) it is possible to acquire and reconstruct the information concerning position and arrival time of photons.

An example of the achievable performance is reported in Figure 11b, in which the head of a Light Emission Diode (LED) was focus (trough a set of lens) on the top, alternative, of ccd/SPAD-array surface. The image was reconstructed after the subtraction of the dark count rate of each pixel. To maximize the image contrast we fix the black level of the colour scale at counting rate less than $500 \mathrm{~Hz}$. 


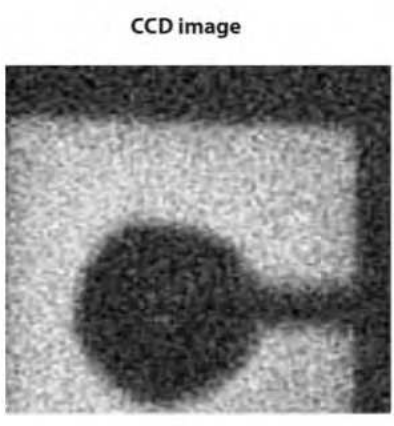

a)

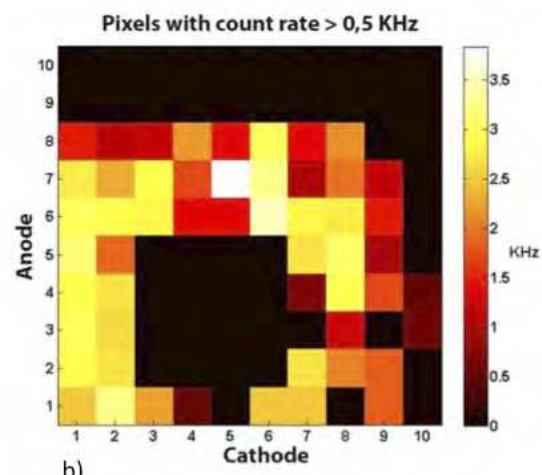

b)

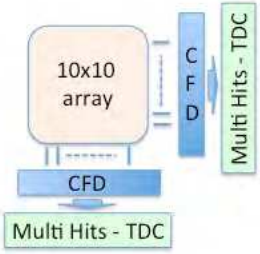

c)

Fig. 11. a) CCD image from the Light Emission Diode. $b$ ) The same image obtained by 10x10 array. c) Schema Readout electronic.

The possibility to adjust the photon flux with a fine precision is a fundamental requirement in order to characterize a TRC. The use of LED source guarantees this possibility through the current limitation on it. With this regulation capability it is possible to collect image with a normal CCD camera (fig. 11a) at medium photon rate and test the SPAD matrix capability in all the sensor dynamic range, from single photon up to the saturation level. By using time modulated current signals it is also possible to test the time dependent characteristic of whole system.

TRC doesn't have frame time limitations like frame duration, frame rate, minimum time distance between frames etc. Every collected photons give his contribution to a continuous streaming of the photon source, the pictures can extracted putting together all the photons during a defined time, or can be collected in groups forming a sequence of video frames without any dead time between frames. The time and spatial positions of all detected photons are collected, and can be used for many correlation technique that use time and/or spatial correlation analysis.

\subsubsection{Current status and prospective}

The solution sketched in figure $9 \mathrm{~b}$ is very promising. Arrays of several size and configuration (up to 170x170 elements) has been designed and manufactured in collaboration with FBK-IRST and are currently under test a characterization.

As previous mentioned, this solution guarantees the collection of the total OR signal (like in SiPM configuration) simplifying the ancillary readout circuitry. From the back, through the anodic contact, you can extract the photon arrival time information (e.g. through a single channel of Multi-Hit TDC) while the position information can be extracted from the front by using, for example, the scheme reported in figure $12 \mathrm{~b}$.

An alternative readout strategy currently under development is reported in figure 12a. A resistive path is realised on the row contacts and column contacts. By using the charge repartition principle it is possible to determine the row and column fired just sampling the 
two signals at the end of the chain. This solution drastically reduces the number of reading channels up to two for rows and two for columns.

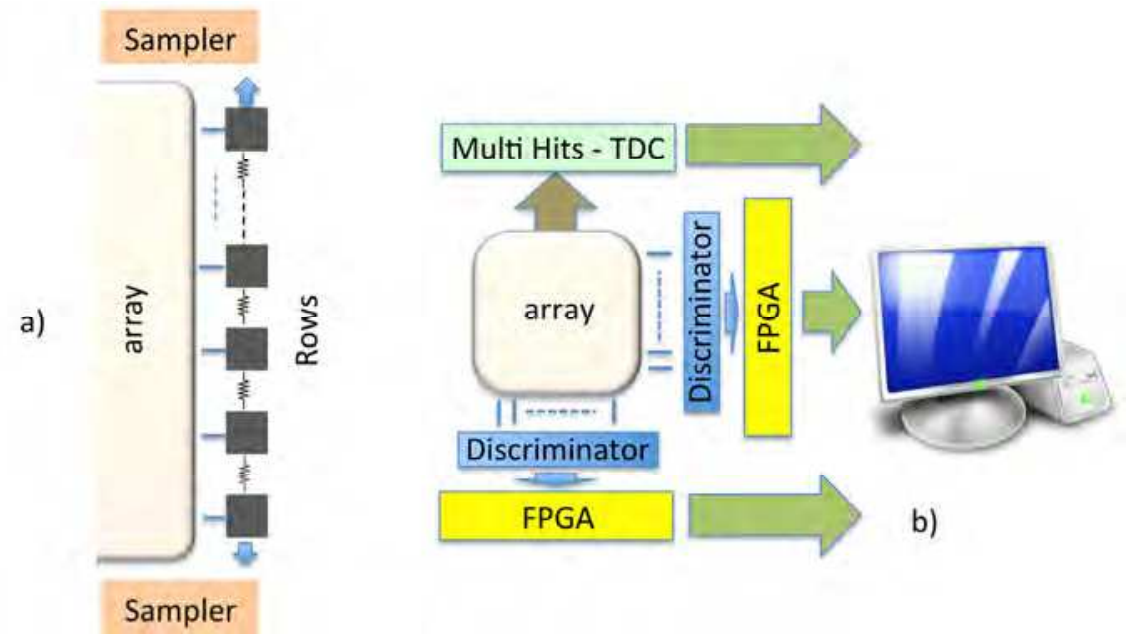

Fig. 12. a) Sketch of the alternative readout strategy through the rows resistive path and signals sampling. $b$ ) Possible readout schema of fig. $9 \mathrm{~b}$ array.

\section{Conclusion}

In the last years, the $R \& D$ of SPADs technology has proceeded at great sped. The main challenge towards imaging devices is geometry and process optimization to yield high compactness while not excessively degrading performance.

On the other hand to make a Time Resolved Camera a compact object available on the market, many efforts have still to be made, in terms of identifying the best readout strategies and ancillary circuitries.

\section{Acknowledgments}

The author would like to acknowledge the partners of the collaboration, ST-Microelectronics and FBK-IRST and in particular G. Fallica and C. Piemonte and the $5^{\text {th }}$ National Committee of INFN that funded this activity.

\section{References}

Jares-Erijman, E. A. and Jovin, T. M. (2003). FRET imaging, Nat. Biotechnol. Vol. 21 pp. 13871395, ISSN 1087-0156

Becker, W. et al., (2006). Fluorescence lifetime images and correlation spectra obtained by multidimensional time-correlated single photon counting, Microsc. Res. Tech. Vol. 69 pp. 186 - 195, ISSN 1059-910X 
Schwille, P. et al., (1999). Molecular dynamics in living cells observed by fluorescence correlation spectroscopy with one and two-photon excitation, Biophys. J. Vol. 77 pp. 2251 - 2265, ISSN 0006-3495

Strickland, D. et al., (1985). Compression of amplified chirped optica pulses, Opt. Comunic. Vol. 56 pp. 219-221, ISSN 0030-4018

McPhate, J. et al., (2005) Noiseless kilohertz-frame-rate imaging detector based on microchannel plates readout Medipix2 CMOS pixel chip, Proc. SPIE Vol. 5881 pp. 88 -97 ISBN 9780819458865

Etoh, T. G. et al. (2005) Design of the PC-ISIS: photon-counting in-situ storage image sensor, IEEE Workshop on CCDs and Advanced Image Sensors (Karuizawa, Japan) pp. 113-116 Available on from http:/ / www.max.hi-ho.ne.jp/teranishi/

Kawai, N. et al. (2005) A low-noise signal readout circuit using double-stage noise cancelling architecture for CMOS image sensors IEEE Workshop on CCDs and Advanced Image Sensors (Karuizawa, Japan) pp. 27-30 Available on from http://www.max.hiho.ne.jp/teranishi/

Hynecek, J. et al. (2001) Impactron - a new solid state image intensifier, IEEE Trans. on Electron Devices Vol. 48 pp. 2238-41 ISSN 0018-9383

Cova, S. et al. (1981) Towards picosecond resolution with single-photon avalanche diodes, Rev. of Scie. Instrum. Vol. 52 pp. 408-412 ISSN 0034-6748

Cova, S. et al (1983) A semiconductor detector for measuring ultraweak fluorescence decays with 70 ps FWHM resolution, IEEE J. Quantum Electronics Vol. 19 pp. 630-634 ISSN 00189197

Cova, S. et al. (1989) Trapping phenomena in avalanche photodiodes on nanosecond scale, IEEE Electron Device Lett. Vol. 12, pp. 685-687 ISSN 0741-3106

Cova, S. et al. (1996) Avalanche photodiodes and quenching circuits for single photon detection, Appl. Opt. Vol. 35, pp. 1956-1976 ISSN 0003-6935.

Mc Intyre, R.J. (1961) Theory of Microplasma Instability in Silicon, J. Appl. Phys. Vol. 35 pp. 1370 ISSN 0021-8979

Mc Intyre, R.J. (1966) Multiplication noise in uniform avalanche diodes, IEEE Trans. on Electron Devices Vol. 13 pp. 164-168 ISSN 0018-9383

Mc Intyre, R.J. 1985 Recent developments in silicon avalanche photodiodes, Measurement Vol. 3 pp. 146-152 ISSN 0263-2241

Hurkx, G.A.M. et al. (1992) A new analytical diode model including tunneling and avalanche breakdown, IEEE Trans. Electron Devices, Vol. 39, pp ISSN 0018-9383

Oldham, W.G. et al. (1972) Triggering phenomena in avalanche diodes," IEEE Trans. Electron Devices, Vol. 19, pp. 1056-1060 ISSN ISSN 0018-9383

Zappa $\mathrm{F}$ et al. (1997) Integrated array of avalanche photodiodes for single-photon counting, IEEE ESSDERC (Stuttgart, Germany) pp 600-603

Kindt, W.J. (1999) Geiger mode avalanche photodiode arrays for spatially resolved single photon counting, PhD Thesis Delft University Press, ISBN 90-407-1845-8

Sciacca, et. al. (2003) Silicon planar technology for single-photon optical detectors, IEEE Trans. on Elect. Dev. Vol. 50, pp. 918-925 ISSN 0018-9383

Ripamonti, G.et al. (1985) Carrier diffusion effects in the time-response of a fast photodiode, Solid State Electron., Vol. 28, pp. 925-931, ISSN 0038-1101

Lacaita, A. et al. (1989) Double epitaxy improves singlephoton avalanche diode performance, Electron. Lett., Vol. 25, nº 13 ISSN 0013-5194 
Mingguo, L. et al. (2008) Reduce Afterpulsing of single photon avalanche diodes using passive quenching with active reset, IEEE Jour. of Quant. Electr. Vol. 44 n5 1077-260X

Niclass, C et al. (2005) Design and characterization of a CMOS 3-D image sensor based on single photon avalanche diodes, IEEE J. Solid State Circuits Vol. 40 pp. 1847-1854 ISSN 00189200

Neri, L. et al. (2010) Generalization of DT equations for time dipendent sources, Sensors Vol. 10, pp. 10828-10836 ISSN 1424-8220

Mazzillo, M. et al. (2008) Single-photon avalanche photodiodes with integrated quenching resistor, Nucl. Instr. Metho. A Vol. 591 pp. 367-373 ISSN 0168-9002

Tyagi, M.S. (1991) Introduction to Semiconductor Materials and Devices, Wiley, ISBN 0471605603 9780471605607, New York.

Grant, W.N et al. (1973) Impact Ionization or Ionization Breakdown Gain and Coefficient Calculator Solid-state Electron. Vol. 16 pp. 1189 ISSN 0038-1101

Bonanno, G. et al (2005) Electro-Optical Characteristics of the Single Photon Avalanche Diode (SPAD), Scientific Detectors for Astronomy, pp. 461 ISBN-1-4020-4329-5 Springer, Berlin, Dordrecht.

Privetara, S. et al. (2008) Single Photon Avalanche Diodes: towards the large bidimensional arrays, Sensors, Vol. 8 pp. 4636-4655 ISSN 1424-8220

Niclass, C. et al. (2005) A single photon detector array with $64 \times 64$ resolution and millimetric depth accuracy for 3D imaging, IEEE Int. Solid-State Circuits Conf. (ISSCC) (San Francisco, USA) pp 364-365

Niclass, C et al. (2006) A $64 \times 48$ single photon avalanche diode array with event-driven readout, Eur. Solid-State Circuits Conf. (ESSCIRC), Montreaux, Switzerland.

Tudisco, S. et al. (2009) Bi-dimensional arrays of SPAD for time-resolved single photon imaging, Nucl. Instr. Method. A Vol. 610 pp. 138-141 ISSN: 0168-9002 


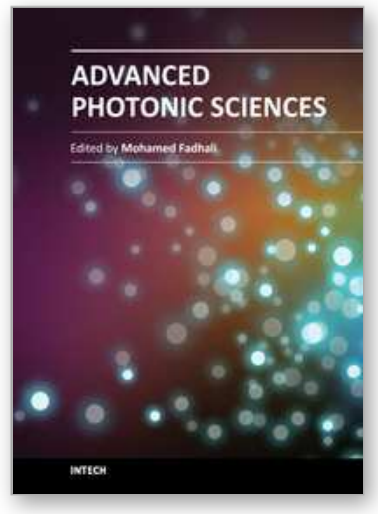

\author{
Advanced Photonic Sciences \\ Edited by Dr. Mohamed Fadhali
}

ISBN 978-953-51-0153-6

Hard cover, 374 pages

Publisher InTech

Published online 21, March, 2012

Published in print edition March, 2012

The new emerging field of photonics has significantly attracted the interest of many societies, professionals and researchers around the world. The great importance of this field is due to its applicability and possible utilization in almost all scientific and industrial areas. This book presents some advanced research topics in photonics. It consists of 16 chapters organized into three sections: Integrated Photonics, Photonic Materials and Photonic Applications. It can be said that this book is a good contribution for paving the way for further innovations in photonic technology. The chapters have been written and reviewed by well-experienced researchers in their fields. In their contributions they demonstrated the most profound knowledge and expertise for interested individuals in this expanding field. The book will be a good reference for experienced professionals, academics and researchers as well as young researchers only starting their carrier in this field.

\title{
How to reference
}

In order to correctly reference this scholarly work, feel free to copy and paste the following:

Salvatore Tudisco (2012). Time Resolved Camera: The New Frontier of Imaging Devices, Advanced Photonic Sciences, Dr. Mohamed Fadhali (Ed.), ISBN: 978-953-51-0153-6, InTech, Available from: http://www.intechopen.com/books/advanced-photonic-sciences/time-resolved-camera-the-new-frontier-ofimaging-devices

\section{INTECH}

open science | open minds

\author{
InTech Europe \\ University Campus STeP Ri \\ Slavka Krautzeka 83/A \\ 51000 Rijeka, Croatia \\ Phone: +385 (51) 770447 \\ Fax: +385 (51) 686166 \\ www.intechopen.com
}

\author{
InTech China \\ Unit 405, Office Block, Hotel Equatorial Shanghai \\ No.65, Yan An Road (West), Shanghai, 200040, China \\ 中国上海市延安西路65号上海国际贵都大饭店办公楼 405 单元 \\ Phone: +86-21-62489820 \\ Fax: +86-21-62489821
}


(C) 2012 The Author(s). Licensee IntechOpen. This is an open access article distributed under the terms of the Creative Commons Attribution 3.0 License, which permits unrestricted use, distribution, and reproduction in any medium, provided the original work is properly cited. 